PSI-PR-11-02

Phys. Lett. A 375 (2011) 3781

\title{
A New Path-Integral Representation of the T-Matrix in Potential Scattering
}

\author{
J. Carron ${ }^{1}$ and R. Rosenfelder ${ }^{2}$ \\ ${ }^{1}$ Institute for Astronomy, ETH Zurich, Wolfgang-Pauli-Strasse 27, CH-8093 Zürich, Switzerland \\ ${ }^{2}$ Particle Theory Group, Paul Scherrer Institute, CH-5232 Villigen PSI, Switzerland
}

\begin{abstract}
We employ the method used by Barbashov and collaborators in Quantum Field Theory to derive a pathintegral representation of the $T$-matrix in nonrelativistic potential scattering which is free of functional integration over fictitious variables as was necessary before. The resulting expression serves as a starting point for a variational approximation applied to high-energy scattering from a Gaussian potential. Good agreement with exact partial-wave calculations is found even at large scattering angles. A novel path-integral representation of the scattering length is obtained in the low-energy limit.
\end{abstract}


1. Path integral methods are not only useful in bound-state problems but also give new insights and approximation schemes in quantum scattering. Recently two path-integral representations for the $T$-matrix in nonrelativistic potential scattering have been derived in Ref. 1] starting from the $S$-matrix as (infinite) limit of the time evolution operator in the interaction picture. For a nonrelativistic particle of mass $m$ moving in a potential $V(\mathbf{x})$ they take the form

$$
\mathcal{T}_{i \rightarrow f}=i \frac{K}{m} \int d^{2} b e^{-i \mathbf{q} \cdot \mathbf{b}}[S(\mathbf{b})-1]
$$

where

$$
\mathbf{K}=\frac{1}{2}\left(\mathbf{k}_{i}+\mathbf{k}_{f}\right), K \equiv|\mathbf{K}|=k \cos \frac{\theta}{2}, \quad \mathbf{q}=\mathbf{k}_{f}-\mathbf{k}_{i}, q \equiv|\mathbf{q}|=2 k \sin \frac{\theta}{2}
$$

are the mean momentum and momentum transfer, respectively. $E=k^{2} /(2 m)$ is the scattering energy (we set $\hbar=1)$ and $\theta$ the scattering angle.

The main features of these representations are functional integrations over velocities without boundary conditions and the use of "phantom" degrees of freedom to get rid of explicit phases which would diverge in the limit of large scattering times. Two versions exist which are distinguished by the reference path along which the particle dominantly travels and the dimensionality $d$ of the "anti-velocity" $\mathbf{w}(t)$ which is needed to achieve the cancellation 1 .

$$
\begin{aligned}
S(\mathbf{b}) & =\int \mathcal{D}^{3} v \mathcal{D}^{d} w \exp \left\{i \int_{-\infty}^{+\infty} d t \frac{m}{2}\left[\mathbf{v}^{2}(t)-\mathbf{w}^{2}(t)\right]\right\} e^{i \chi(\mathbf{b}, \mathbf{v}, \mathbf{w}]} \\
\chi(\mathbf{b}, \mathbf{v}, \mathbf{w}] & =\int_{-\infty}^{+\infty} d t V\left(\mathbf{b}+\mathbf{x}_{\text {ref }}(t)+\mathbf{x}_{\text {quant }}(t, \mathbf{v}, \mathbf{w}]\right)
\end{aligned}
$$

In the first case

$$
\mathbf{x}_{\mathrm{ref}}^{(d=3)}(t)=\frac{\mathbf{K}}{m} t, \quad \mathbf{x}_{\text {quant }}^{(d=3)}(t, \mathbf{v}, \mathbf{w}]=\mathbf{x}_{\mathbf{v}}(t)-\mathbf{x}_{\mathbf{w}}(0)
$$

the reference path is a straight-line path along the mean momentum and

$$
\mathbf{x}_{v}(t)=\frac{1}{2} \int_{-\infty}^{+\infty} d t^{\prime} \operatorname{sgn}\left(t-t^{\prime}\right) \mathbf{v}\left(t^{\prime}\right), \dot{\mathbf{x}}_{v}(t)=\mathbf{v}(t)
$$

describes the quantum fluctuations, $\operatorname{sgn}(x)=2 \Theta(x)-1$ is the sign-function. In the second case the anti-velocity is only 1-dimensional

$$
\mathbf{x}_{\text {ref }}^{(d=1)}(t) \equiv \mathbf{x}_{\text {ray }}(t)=\left[\frac{\mathbf{k}_{i}}{m} \Theta(-t)+\frac{\mathbf{k}_{f}}{m} \Theta(t)\right] t, \quad \mathbf{x}_{\text {quant }}^{(d=1)}(t, \mathbf{v}, w]=\mathbf{x}_{\mathbf{v}}(t)-\mathbf{x}_{\perp \mathbf{v}}(0)-\hat{\mathbf{K}} x_{\| w}(0)
$$

and the reference path is a ray along the initial momentum for $t<0$ and along the final momentum for $t>0$. In Ref. [1] it has been shown that both these representations reproduce the Born series to all orders if expanded in powers of the potential.

Is it possible to get rid of the "anti-velocity" altogether? In the present Letter we show that this is feasible by using a method which has been introduced by Barbashov and coworkers [2, 3, 4, nearly 40 years ago. They showed how one can amputate exact Green functions in Quantum Field Theory without using perturbation theory but their work doesn't seem to have received much attention apart from the (rather specialized) field of quantum gravity where Fabbrichesi, Han and others [5, 6, 7, , 8, have extensively used the Barbashov method to describe gravitational scattering. Our main aim in this work is to transcribe their method to the nonrelativistic

\footnotetext{
${ }^{1}$ Here and in the following the path integrals are normalized such that the free (Gaussian) integral is unity. Our notation indicates that $\chi$ is a function of the impact parameter $\mathbf{b}$ but a functional of $\mathbf{v}(t)$ and $\mathbf{w}(t)$. Similarly for $\mathbf{x}_{\text {quant }}$.
} 
case and to use the result for an approximate variational calculation similar to the one investigated in Refs. [9, 10]. As a by-product a new path-integral representation of the scattering length is obtained.

2. Our starting point is the following "reduction formula" for obtaining the on-shell $T$-matrix from the full Green function

$$
\left.\left\langle\mathbf{k}_{f}|\hat{T}| \mathbf{k}_{i}\right\rangle\right|_{\mathbf{k}_{i}^{2}=\mathbf{k}_{f}^{2}=2 m E}=\lim _{\mathbf{k}_{i}^{2}, \mathbf{k}_{f}^{2} \rightarrow 2 m E}\left\langle\mathbf{k}_{f}\left|\left(E-\hat{H}_{0}\right) \hat{G}(E)\left(E-\hat{H}_{0}\right)\right| \mathbf{k}_{i}\right\rangle .
$$

This is easily proved by using the definition of the exact Green function

$$
\hat{G}(E):=\frac{1}{E-\hat{H}+i 0}=\frac{1}{E-\hat{H}_{0}-\hat{V}+i 0}
$$

and some standard algebraic manipulations:

$$
\begin{aligned}
\left(E-\hat{H}_{0}\right) \hat{G}(E)\left(E-\hat{H}_{0}\right) & =(E-\hat{H}+\hat{V}) \hat{G}(E)\left(E-\hat{H}_{0}\right)=(1+\hat{V} \hat{G}(E))(E-\hat{H}+\hat{V}) \\
& =E-\hat{H}_{0}+\hat{V}(1+\hat{G}(E) \hat{V}) \equiv E-\hat{H}_{0}+\hat{T} .
\end{aligned}
$$

When going to the energy shell the first term on the r.h.s. of Eq. (10) vanishes and we obtain indeed the matrix element of the $T$-matrix between initial and final momentum states. Eq. (8) is the nonrelativistic counterpart of the standard Lehmann-Symanzik-Zimmermann (LSZ) reduction formula given in any textbook on Quantum Field Theory (see, e.g. Eq. (7.42) in Ref. [11]). As other tools are available, this relation seems not to be employed in standard nonrelativistic scattering theory. One exception is Ref. [12] where the time-dependent version in Eq. (16.82) is used to illustrate the "close analogy" of the reduction formula "with the $S$ matrix constructed in propagator theory".

Assuming that the interaction $\hat{V}$ is given by a local potential $V(\hat{\mathbf{x}})$ we now employ the Schwinger representation of the full Green function (9)

$$
\hat{G}(E)=-i \int_{0}^{\infty} d T e^{i(E-\hat{H}+i 0) T}
$$

and the velocity path integral for the time evolution operator as given in Eq. (4) of Ref. 1]. Then one obtains from Eq. (8) 2

$$
\begin{aligned}
\mathcal{T}_{i \rightarrow f} & =(-i) \lim _{\mathbf{k}_{i}^{2}, \mathbf{k}_{f}^{2} \rightarrow 2 m E}\left(E-\frac{\mathbf{k}_{i}^{2}}{2 m}\right)\left(E-\frac{\mathbf{k}_{f}^{2}}{2 m}\right) \int_{0}^{\infty} d T e^{i E T} \int d^{3} x d^{3} x^{\prime} e^{-i \mathbf{k}_{f} \cdot \mathbf{x}^{\prime}+i \mathbf{k}_{i} \cdot \mathbf{x}} \\
& \times \int \mathcal{D}^{3} v \delta^{(3)}\left(\mathbf{x}^{\prime}-\mathbf{x}-\int_{0}^{T} d \tau \mathbf{v}(\tau)\right) \exp \left\{i \int_{0}^{T} d t\left[\frac{m}{2} \mathbf{v}^{2}(t)-V\left(\mathbf{x}+\int_{0}^{t} d \tau \mathbf{v}(\tau)\right)\right]\right\} .
\end{aligned}
$$

Performing the $\mathbf{x}^{\prime}$-integration and shifting the velocity $\mathbf{v}(\tau)=\overline{\mathbf{v}}(\tau)+\mathbf{k}_{f} / m$ yields

$$
\begin{aligned}
\mathcal{T}_{i \rightarrow f}= & (-i) \lim _{\Delta_{i}, \Delta_{f} \rightarrow 0}\left(\Delta_{i} \Delta_{f}\right) \cdot \int_{0}^{\infty} d T e^{i \Delta_{f} T} \int d^{3} x e^{-i\left(\mathbf{k}_{f}-\mathbf{k}_{i}\right) \cdot \mathbf{x}} \\
& \times \int \mathcal{D}^{3} \bar{v} \exp \left\{i \int_{0}^{T} d t\left[\frac{m}{2} \overline{\mathbf{v}}^{2}(t)-V\left(\mathbf{x}+\frac{\mathbf{k}_{f}}{m} t+\int_{0}^{t} d \tau \overline{\mathbf{v}}(\tau)\right)\right]\right\}
\end{aligned}
$$

\footnotetext{
${ }^{2}$ Our scattering states are normalized to $\langle f \mid i\rangle=(2 \pi)^{3} \delta^{(3)}\left(\mathbf{k}_{f}-\mathbf{k}_{i}\right)$.
} 
where

$$
\Delta_{i}:=E-\frac{\mathbf{k}_{i}^{2}}{2 m}, \quad \Delta_{f}:=E-\frac{\mathbf{k}_{f}^{2}}{2 m}
$$

is the initial or final off-shellness. It is obvious that Eq. (13) is only non-zero if the Fourier-transformed Green function develops two poles when $\mathbf{k}_{i}^{2}, \mathbf{k}_{f}^{2} \rightarrow 2 m E$. How is that possible? It only can originate from the integral over $T$, presumably from $T \rightarrow \infty$ (one intuitively expects that the particle becomes on-(energy-)shell for infinite times). Indeed for any smooth function $f(T)$ one has

$$
\begin{aligned}
-i \lim _{\Delta \rightarrow 0} \Delta \int_{0}^{\infty} d T e^{i(\Delta+i 0) T} f(T) & =\quad-\lim _{\Delta \rightarrow 0} \int_{0}^{\infty} d T \frac{\partial}{\partial T}\left\{e^{i(\Delta+i 0) T}\right\} f(T) \\
& \stackrel{(\text { partial int.) }}{=} \\
& f(0)+\lim _{\Delta \rightarrow 0} \int_{0}^{\infty} d T e^{i \Delta T} \frac{\partial f(T)}{\partial T}=f(\infty) .
\end{aligned}
$$

However, integration over $T$ only takes away the factor $\Delta_{f}$ but not the other factor $\Delta_{i}$ and one obviously needs a second integration. The main trick in Barbashov's treatment is a way to provide for this second time integration by first subtracting from Eq. (13) a term (a "1") which does not contribute in the on-shell limit

$$
\begin{aligned}
\mathcal{T}_{i \rightarrow f}= & -i \lim _{\Delta_{i}, \Delta_{f} \rightarrow 0} \Delta_{i} \Delta_{f} \int_{0}^{\infty} d T e^{i\left(\Delta_{f}+i 0\right) T} \int d^{3} x e^{-i \mathbf{q} \cdot \mathbf{x}} \\
& \times \int \mathcal{D}^{3} v \exp \left(i \int_{0}^{T} d t \frac{m}{2} \mathbf{v}^{2}(t)\right)\left\{\exp \left[-i \int_{0}^{T} d t V\left(\boldsymbol{\rho}_{f}(\mathbf{x}, t, \mathbf{v}]\right)\right]-1\right\} .
\end{aligned}
$$

This is because the free Green function is proportional to $\delta^{(3)}\left(\mathbf{k}_{f}-\mathbf{k}_{i}\right) /\left(E-\mathbf{k}_{i}^{2} /(2 m)\right)$. We use the abbreviation

$$
\boldsymbol{\rho}_{f}(\mathbf{x}, t, \mathbf{v}]:=\mathbf{x}+\frac{\mathbf{k}_{f}}{m} t+\int_{0}^{t} d \tau \mathbf{v}(\tau)
$$

and omit the bar on the velocity variable. Now the factor in curly brackets in Eq. (16) can be written as

$$
\exp \left[-i \int_{0}^{T} d t V\left(\boldsymbol{\rho}_{f}(\mathbf{x}, t, \mathbf{v}]\right)\right]-1=\left[-i \int_{0}^{T} d \xi V\left(\boldsymbol{\rho}_{f}(\mathbf{x}, \xi, \mathbf{v}]\right)\right] \int_{0}^{1} d \lambda \exp \left[-i \lambda \int_{0}^{T} d t V\left(\boldsymbol{\rho}_{f}(\mathbf{x}, t, \mathbf{v}]\right)\right]
$$

which provides an additional integration over the time $\xi$.

3. Since the algebra in Ref. [6] (which we follow with several corrections) is somewhat sketchy and involved we list in more detail the steps to be taken in the following:

(i) Write

$$
\int_{0}^{\infty} d T \int_{0}^{T} d \xi \ldots=\int_{0}^{\infty} d \xi \int_{\xi}^{\infty} d T \ldots
$$

and substitute $T=: \xi+s$ so that $s \in[0, \infty]$. We then have

$$
\begin{aligned}
\mathcal{T}_{i \rightarrow f}= & (-i)^{2} \lim _{\Delta_{i}, \Delta_{f} \rightarrow 0} \Delta_{i} \Delta_{f} \int_{0}^{\infty} d \xi \int_{0}^{\infty} d s e^{i\left(\Delta_{f}+i 0\right)(\xi+s)} \int \mathcal{D}^{3} v \exp \left(i \int_{0}^{\xi+s} d t \frac{m}{2} \mathbf{v}^{2}(t)\right) \\
& \times \int d^{3} x e^{-i \mathbf{q} \cdot \mathbf{x}} V\left(\boldsymbol{\rho}_{f}(\mathbf{x}, \xi, \mathbf{v}]\right) \int_{0}^{1} d \lambda \exp \left[-i \lambda \int_{0}^{\xi+s} d t V\left(\boldsymbol{\rho}_{f}(\mathbf{x}, t, \mathbf{v}]\right)\right]
\end{aligned}
$$

ii) Substitute

$$
\overline{\mathbf{x}}=\boldsymbol{\rho}_{f}(\mathbf{x}, \xi, \mathbf{v}]=\mathbf{x}+\frac{\mathbf{k}_{f}}{m} \xi+\int_{0}^{\xi} d \tau \mathbf{v}(\tau)
$$


Collecting the pieces in the exponents we then obtain

$$
\begin{aligned}
\mathcal{T}_{i \rightarrow f}= & (-i)^{2} \lim _{\Delta_{i}, \Delta_{f} \rightarrow 0} \Delta_{i} \Delta_{f} \int_{0}^{\infty} d \xi \exp \left[i\left(\Delta_{i}+i 0\right) \xi+i \underline{\underline{\mathbf{q}^{2}}} \xi\right] \\
& \times \int_{0}^{\infty} d s \exp \left[i\left(\Delta_{f}+i 0\right) s\right] \int \mathcal{D}^{3} v \exp \left[i \int_{0}^{\xi+s} d \tau \frac{m}{2} \mathbf{v}^{2}(\tau)+i \mathbf{q} \cdot \int_{0}^{\xi} d \tau \mathbf{v}(\tau)\right] \\
& \times \int d^{3} \overline{\mathbf{x}} e^{-i \mathbf{q} \cdot \overline{\mathbf{x}}} V(\overline{\mathbf{x}}) \int_{0}^{1} d \lambda \exp \left[-i \lambda \int_{0}^{\xi+s} d t V\left(\overline{\mathbf{x}}+\boldsymbol{\rho}_{f}(\overline{\mathbf{x}}, t, \mathbf{v}]-\boldsymbol{\rho}_{f}(\overline{\mathbf{x}}, \xi, \mathbf{v}]\right)\right]
\end{aligned}
$$

(iii) The unwanted pieces (underlined in Eq. (22)) are eliminated by yet another shift

$$
\overline{\mathbf{v}}(\tau)=\mathbf{v}(\tau)+\frac{\mathbf{q}}{m} \Theta(\xi-\tau) .
$$

The argument in the last potential term then becomes

$$
\overline{\mathbf{x}}+\boldsymbol{\rho}_{f}(\overline{\mathbf{x}}, t, \mathbf{v}]-\boldsymbol{\rho}_{f}(\overline{\mathbf{x}}, \xi, \mathbf{v}]=\overline{\mathbf{x}}+\left[\frac{\mathbf{k}_{f}}{m} \Theta(t-\xi)+\frac{\mathbf{k}_{i}}{m} \Theta(\xi-t)\right](t-\xi)+\int_{\xi}^{t} d \tau \overline{\mathbf{v}}(\tau) .
$$

Finally, we define

$$
\bar{\tau}:=\tau-\xi, \quad \bar{t}:=t-\xi, \quad \overline{\overline{\mathbf{v}}}(\bar{\tau}):=\overline{\mathbf{v}}(\bar{\tau}+\xi)
$$

so that the argument (24) becomes

$$
\overline{\mathbf{x}}+\left[\frac{\mathbf{k}_{f}}{m} \Theta(\bar{t})+\frac{\mathbf{k}_{i}}{m} \Theta(-\bar{t})\right] \bar{t}+\int_{0}^{\bar{t}} d \bar{\tau} \overline{\overline{\mathbf{v}}}(\bar{\tau})=: \boldsymbol{\rho}_{\text {ray }}(\overline{\mathbf{x}}, \bar{t}, \overline{\overline{\mathbf{v}}}] .
$$

Omitting the bars over the transformed variables we thus have

$$
\begin{aligned}
\mathcal{T}_{i \rightarrow f}= & (-i)^{2} \lim _{\Delta_{i}, \Delta_{f} \rightarrow 0} \Delta_{i} \Delta_{f} \int_{0}^{\infty} d \xi e^{i\left(\Delta_{i}+i 0\right) \xi} \int_{0}^{\infty} d s e^{i\left(\Delta_{f}+i 0\right) s} \int d^{3} x e^{-i \mathbf{q} \cdot \mathbf{x}} V(\mathbf{x}) \\
& \times \int \mathcal{D}^{3} v \exp \left[i \int_{-\xi}^{s} d \tau \frac{m}{2} \mathbf{v}^{2}(\tau)\right] \int_{0}^{1} d \lambda \exp \left[-i \lambda \int_{-\xi}^{s} d t V\left(\boldsymbol{\rho}_{\text {ray }}(\mathbf{x}, t, \mathbf{v}]\right)\right] .
\end{aligned}
$$

It is now possible to truncate both external legs by using Eq. (15) and to obtain the final result

$$
\mathcal{T}_{i \rightarrow f}=\int d^{3} x e^{-i \mathbf{q} \cdot \mathbf{x}} V(\mathbf{x}) \int \mathcal{D}^{3} v \exp \left[i \int_{-\infty}^{+\infty} d t \frac{m}{2} \mathbf{v}^{2}(t)\right] \int_{0}^{1} d \lambda \exp \left[-i \lambda \int_{-\infty}^{+\infty} d t V\left(\boldsymbol{\rho}_{\text {ray }}(\mathbf{x}, t, \mathbf{v}]\right)\right] .
$$

Of course, the $\lambda$-integration can be performed trivially but for many applications it is better to leave it in this form (see below).

Note that in the second potential term the particle propagates mainly along a ray formed by incident and outgoing momenta exactly as in the "ray" representation (7) while the quantum fluctuations are described by the functional integration over the velocity. Indeed

$$
\rho_{\text {ray }}(\mathbf{x}, t, \mathbf{v})=\mathbf{x}+\mathbf{x}_{\text {ray }}(t)+\mathbf{x}_{\mathbf{v}}(t)-\mathbf{x}_{\mathbf{v}}(0) .
$$

Eq. (28) also gives a precise definition for a local "pseudopotential" (in general, this is a nonlocal object; see, e.g. chapter 6.2 in Ref. [13) whose Fourier transform yields the exact $T$-matrix. 
While it is not possible to set $k=0$ directly in Eq. (11) the representation (28) allows that without impunity. Therefore, defining the scattering length $a$ as the negative $(k \rightarrow 0)$-limit of the scattering amplitude $f=-m \mathcal{T} /(2 \pi)$ (as in Eq. (X.47) of Ref. [14]) we immediately obtain a path-integral representation of the scattering length

$$
a=\frac{m}{2 \pi} \int d^{3} x V(\mathbf{x}) \int \mathcal{D}^{3} v \exp \left[i \int_{-\infty}^{+\infty} d t \frac{m}{2} \mathbf{v}^{2}(t)\right] \int_{0}^{1} d \lambda \exp \left[-i \lambda \int_{-\infty}^{+\infty} d t V\left(\mathbf{x}+\int_{0}^{t} d \tau \mathbf{v}(\tau)\right)\right]
$$

Unfortunately, we do not have a simple argument why the scattering length should be real in potential scattering, i.e. why the imaginary part of the r.h.s of Eq. (30) vanishes. This is one of the shortcomings of a path-integral representation of scattering; others are that basic properties like unitarity (and therefore the optical theorem) are more evident in the traditional operator formulation of Quantum Mechanics.

4. We can use our result as a starting point for new approximation schemes. For example, following Refs. [9. 10] we may variationally approximate the velocity path integral in Eq. (28)

$$
F(\mathbf{x}, \lambda, S]:=\int \mathcal{D}^{3} v e^{i S(\mathbf{x}, \lambda, \mathbf{v}]}, \quad S(\mathbf{x}, \lambda, \mathbf{v}]=\int_{-\infty}^{+\infty} d t\left[\frac{m}{2} \mathbf{v}^{2}(t)-\lambda V\left(\rho_{\text {ray }}(\mathbf{x}, t, \mathbf{v}]\right)\right]
$$

by means of the Feynman-Jensen variational principle. The most general quadratic trial action, which allows the analytic evaluation of the needed path integrals, is

$$
S_{t}[\mathbf{v}]=\frac{m}{2} \mathbf{v}^{T} \mathbb{A} \mathbf{v}+\mathbb{B} \mathbf{v}
$$

in the condensed notation of Ref. [10] where summation over discrete and continuous indices is implied. Evaluation of averages and derivation of the variational equations for the functions $A_{i j}\left(t, t^{\prime}\right), B_{i}(t)$ follow the same lines as in Refs. [9, 10] where more details can be found. Here we just give the results: with this trial action the variational approximation to the $T$-matrix with no anti-velocity reads

$$
\mathcal{T}_{i \rightarrow f}^{(A B 30)}=\int d^{3} x e^{-i \mathbf{q} \cdot \mathbf{x}} V(\mathbf{x}) \int_{0}^{1} d \lambda F_{\text {var }}(\mathbf{x}, \lambda), \quad F_{\text {var }}(\mathbf{x}, \lambda)=e^{i X_{0}+i X_{1}-\Omega}
$$

with

$$
\begin{aligned}
X_{0} & =-\int_{-\infty}^{+\infty} d t U(t) \\
X_{1} & =\frac{1}{2} \nabla U \Sigma_{0} \nabla U \\
\left(\Sigma_{0}\right)_{i j}\left(t, t^{\prime}\right) & =-\frac{\delta_{i j}}{2 m}\left[\left|t-t^{\prime}\right|-\left|t^{\prime}\right|-|t|\right] \\
\Omega & =-\frac{1}{2} \operatorname{Tr}[\ln (1+\Sigma H)-\Sigma H] .
\end{aligned}
$$

Here $U(t)$ is the Gaussian transform of the potential $\lambda V$, i.e.

$$
U(t)=\int \frac{d^{3} p}{(2 \pi)^{3}} \lambda \tilde{V}(\mathbf{p}) \exp \left(i \mathbf{p} \cdot \boldsymbol{\rho}_{\text {var }}(t)-\frac{i}{2} \mathbf{p}^{T} \sigma(t) \mathbf{p}\right), \quad \text { with } \quad \tilde{V}(\mathbf{p})=\int d^{3} x e^{-i \mathbf{p} \cdot \mathbf{x}} V(\mathbf{x})
$$

and $H_{i j}:=\partial_{i} \partial_{j} U$ its Hessian. The width of the Gaussian transform is given by the diagonal value of the matrix $\Sigma$, i.e. $\sigma_{i j}(t)=\Sigma_{i j}(t, t)$. Varying with respect to the variational functions $\mathbb{A}, \mathbb{B}$ leads to variational equations for the trajectory and the (off-diagonal) width:

$$
\begin{aligned}
\boldsymbol{\rho}_{\mathrm{var}}(t) & =\mathbf{x}+\mathbf{x}_{\mathrm{ray}}(t)+\left(\Sigma_{0} \nabla U\right)(t) \\
\Sigma & =\Sigma_{0}+\Sigma_{0} H \Sigma .
\end{aligned}
$$


The first one is a classical equation of motion for the trajectory of the particle as can be seen by differentiating Eq. (35) twice with respect the the time $t$. This gives Newton's equation with a "kick" at $t=0$

$$
m \ddot{\boldsymbol{\rho}}_{\mathrm{var}}(t)=\left(\mathbf{q}+\int_{-\infty}^{+\infty} d t^{\prime} \nabla U\left(t^{\prime}\right)\right) \delta(t)-\nabla U(t) .
$$

The second one is a Lippmann-Schwinger-type equation for the quantum-mechanical spreading of the scattered wave. In the present numerical evaluation of these coupled nonlinear equations we have restricted ourselves to the case where $\mathbb{A}=1$. This amounts to setting the Hessian to zero and to neglect the wave spreading. Then the width of the Gaussian transform is fixed as $\sigma_{i j}(t)=\delta_{i j}|t| / m$ and the quantity $\Omega$ vanishes.

This approximation (B30, in the nomenclature of Ref. [10]) should reproduce the $2^{\text {nd }}$ Born approximation and therefore be roughly as good as the systematic approximations studied in Ref. [10] which included the correction by the second cumulant. Indeed, simple algebra shows that the second Born approximation is contained in our variational approximation: When expanding $\mathcal{T}_{i \rightarrow f}^{(B 30)}$ up to second order in the potential we can neglect the phase $X_{1}$ and set $\exp \left(i X_{0}\right)=1+i X_{0}$. Furthermore the argument of the potential in the phase $X_{0}$ reduces to $\mathbf{x}+\mathbf{x}_{\text {ref }}(t)$ in this order. The $\lambda$-integration is then trivial and with the definition of the Gaussian transformed potential one can perform the $\mathbf{x}$-integration. The result is

$T_{i \rightarrow f}^{(B 30)}=T^{1 \text { st Born }}-\frac{i}{2} \int \frac{d^{3} p_{1} d^{3} p_{2}}{(2 \pi)^{3}} \tilde{V}\left(\mathbf{p}_{1}\right) \tilde{V}\left(\mathbf{p}_{2}\right) \delta^{(3)}\left(\mathbf{p}_{1}+\mathbf{p}_{2}-\mathbf{q}\right) \int_{-\infty}^{+\infty} d t \exp \left[i \mathbf{p}_{2} \cdot \mathbf{x}_{\text {ray }}(t)-\frac{i}{2 m}|t| \mathbf{p}_{2}^{2}\right]+\mathcal{O}\left(V^{3}\right)$

where the Gaussian width in the the "B30"-approximation has been inserted. This allows the $t$-integration to be performed and gives

$$
\begin{aligned}
T_{i \rightarrow f}^{(3-0)}= & T^{1 \mathrm{st} \text { Born }}-\frac{i}{2} \int \frac{d^{3} p_{1} d^{3} p_{2}}{(2 \pi)^{3}} \tilde{V}\left(\mathbf{p}_{1}\right) \tilde{V}\left(\mathbf{p}_{2}\right) \delta^{(3)}\left(\mathbf{p}_{1}+\mathbf{p}_{2}-\mathbf{q}\right) \\
& \times\left[\frac{i}{\mathbf{p}_{2} \cdot \mathbf{k}_{f} / m-\mathbf{p}_{2}^{2} /(2 m)+i 0}-\frac{i}{\mathbf{p}_{2} \cdot \mathbf{k}_{i} / m+\mathbf{p}_{2}^{2} /(2 m)-i 0}\right]+\mathcal{O}\left(V^{3}\right)
\end{aligned}
$$

where the two terms come from the integration over positive and negative times, respectively. Now, putting $\mathbf{p}_{1}=\mathbf{p}-\mathbf{k}_{i}, \quad \mathbf{p}_{2}=\mathbf{k}_{f}-\mathbf{p}$ in the first term and just reversed in the second, one sees that both terms are the same due to energy conservation $E=\mathbf{k}_{i}^{2} /(2 m)=\mathbf{k}_{f}^{2} /(2 m)$ which is already imposed by dealing with the on-shell $T$-matrix. This cancels the factor $1 / 2$ from the $\lambda$-integration so that the result is

$$
\begin{aligned}
T_{i \rightarrow f}^{(B 30)} & =T^{1 \text { st Born }}+\int \frac{d^{3} p}{(2 \pi)^{3}} \tilde{V}\left(\mathbf{k}_{f}-\mathbf{p}\right) \frac{1}{E-\mathbf{p}^{2} /(2 m)+i 0} \tilde{V}\left(\mathbf{p}-\mathbf{k}_{i}\right)+\mathcal{O}\left(V^{3}\right) \\
& =T^{1 \text { st Born }}+\left\langle\mathbf{k}_{f}\left|\hat{V} \frac{1}{E-\hat{H}_{0}+i 0} \hat{V}\right| \mathbf{k}_{i}\right\rangle+\mathcal{O}\left(V^{3}\right) \equiv T^{1 \text { st Born }}+T^{2 \text { nd Born }}+\mathcal{O}\left(V^{3}\right) .
\end{aligned}
$$

We have solved the variational equations by iteration similar as was done in Refs. [9, 10] apart from numerical improvements to speed up the tabulation of the trajectory and to add the asymptotic contribution to integrals with slowly decreasing integrands. Fig. [1 shows the comparison with an exact partial-wave calculation for scattering from a Gaussian potential

$$
V(x)=V_{0} e^{-x^{2} / R^{2}}
$$

at $k R=4,2 m V_{0} R^{2}=-4$ which served as a benchmark calculation in Refs. 9, 10. Plotted is the relative deviation $|\Delta f / f| \equiv\left|\left(f_{\text {var }}-f_{\text {exact }}\right) / f_{\text {exact }}\right|$ and it is seen that our variational approximation outperforms both the first and the second Born approximation. Even at large scattering angles where the cross section is down by many orders of magnitude the deviation is still rather small. 


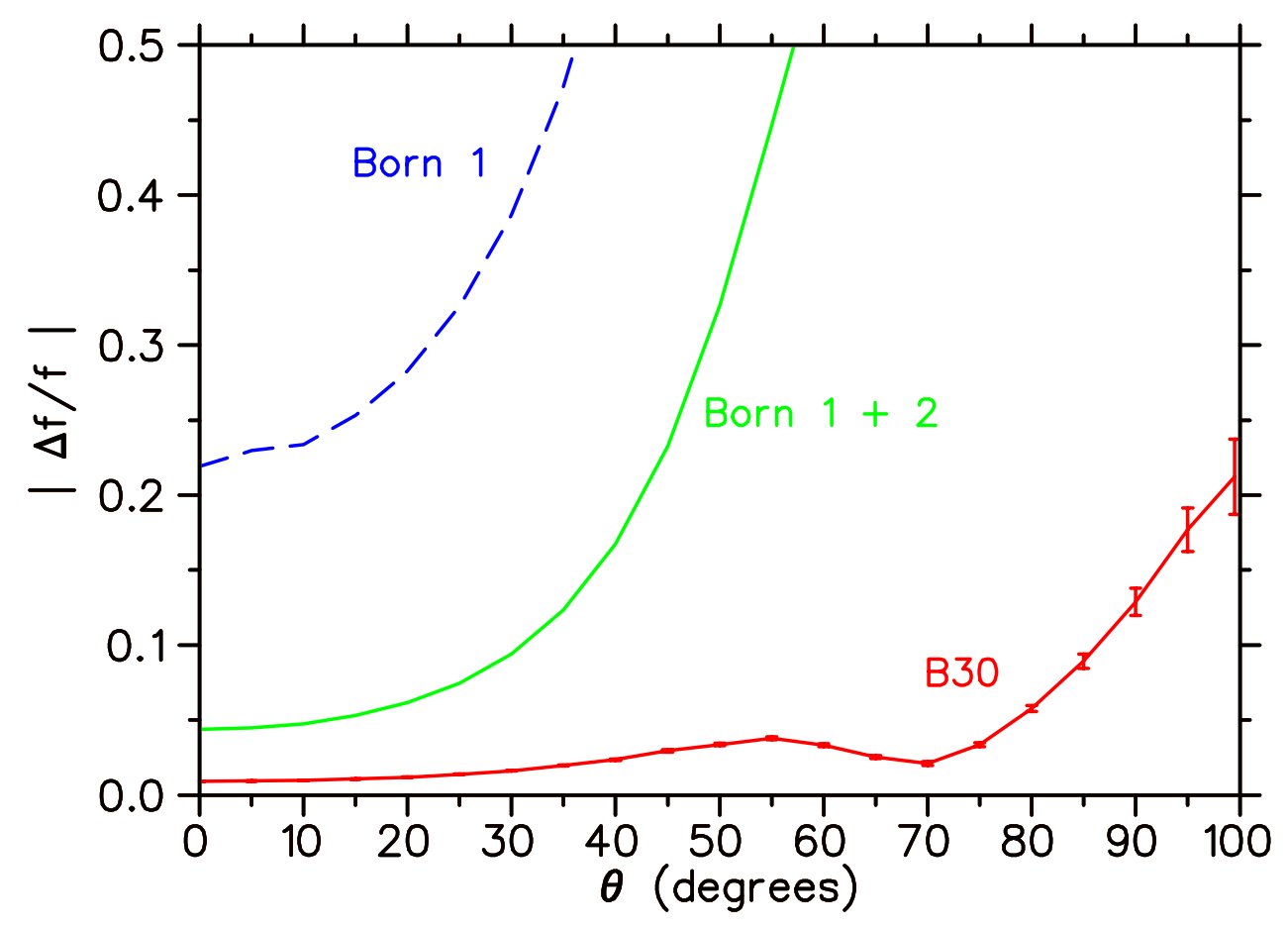

Figure 1: (Color online) The relative deviation $|\Delta f / f|$ of the variational amplitude in the present approximation ("B30") from the exact scattering amplitude as a function of the scattering angle $\theta$. The error bars denote the estimated uncertainty of the numerical evaluation. Also shown are the results from the first and from the first + second Born approximation.

5. In summary we have presented a new path-integral representation for the $T$-matrix in nonrelativistic potential scattering based on an ingenious method for amputating full Green functions in Quantum Field Theory due to Barbashov et al. Compared to previous representations the present one does not need artificial "phantom" degrees of freedom to cancel unphysical contributions.

Of course, there is an (infinite) variety of path-integral representations depending at which level of formal scattering theory one introduces the path integral. For reasons of classification one may count the powers of the potential in front of the velocity path integral. Then the previous forms displayed in Eqs. (11), (4) may be characterized as "level 0"- representations whereas the Barbashov-like result (28) belongs to the "level 1"-class. A path-integral representation based on the formal solution of the Lippmann-Schwinger equation (last line in Eq. (10) ) would lead to a "level 2"-representation and so on. Obviously, higher-level representations become more and more complicated so that the formulations on level zero or one seem to be a good compromise between complexity and efficiency.

As shown by a variational calculation for high-energy scattering from a Gaussian potential the new representation offers new opportunities for approximations, seems to be applicable also at low energy and could be used for stochastic evaluation of real-time scattering similar as in Ref. [15]. At least it is an interesting addition to the standard body of knowledge in nonrelativistic scattering theory originating from field-theoretic methods. 


\section{References}

[1] R. Rosenfelder: "Path Integrals for Potential Scattering," Phys. Rev. A 79 (2009) 012701 arXiv:0806.3217 [nucl-th]].

[2] B. M. Barbashov, S. P. Kuleshov, V. A. Matveev and A. N. Sisakian: "Eikonal approximation in quantumfield theory", Teor. Mat. Fiz. 3 (1970) 342 [English transl.: Theor. Math. Phys. 3 (1970) 555] .

[3] B. M. Barbashov, S. P. Kuleshov, V. A. Matveev, V. N. Pervushin, A. N. Sissakian and A. N. Tavkhelidze: "Straight-line paths approximation for studying high-energy elastic and inelastic hadron collisions in quantum field theory," Phys. Lett. B 33 (1970) 484.

[4] V. A. Matveev and A. N. Tavkhelidze: "On the representation of scattering amplitudes as path integrals in quantum field theory", Teor. Mat. Fiz. 9 (1971) 44 [English transl.: Theor. Math. Phys. 9 (1971) 968] .

[5] M. Fabbrichesi, R. Pettorino, G. Veneziano and G. A. Vilkovisky: "Planckian energy scattering and surface terms in the gravitational action": Nucl. Phys. B 419 (1994) 147.

[6] N. S. Han and E. Ponna: "Straight-line path approximation for studying Planckian-energy scattering in quantum gravity", Nuov. Cim. A 110 (1997) 459.

[7] N. S. Han: "Straight-line path approximation for high-energy elastic and inelastic scattering in quantum gravity" Eur. Phys. J. C 16 (2000) 547.

[8] N. S. Han and N. N. Xuan: "Planckian scattering beyond the eikonal approximation in the functional approach", Eur. Phys. J. C 24 (2002) 643. arXiv:gr-qc/0203054.

[9] J. Carron: "Variational Methods for Path Integral Scattering," arXiv:0903.0273 v2 [nucl-th] .

[10] J. Carron and R. Rosenfelder: "Variational Approximations in a Path-Integral Description of Potential Scattering," Eur. Phys. J. A 45 (2010) 193 [arXiv:0912.4429 [nucl-th]].

[11] M. E. Peskin and D. V. Schroeder: An Introduction to Quantum Field Theory, Addison-Wesley (Reading 1996) .

[12] J. D. Bjorken and S. D. Drell: Relativistic Quantum Fields, McGraw-Hill (New York 1965) .

[13] L. S. Rodberg and R. M. Thaler: Introduction to the Quantum Theory of Scattering, Academic Press (New York 1967).

[14] A. Messiah: Quantum Mechanics, North-Holland (Amsterdam 1965) .

[15] R. Rosenfelder: "Exact Path-Integral Representations for the T-Matrix in Nonrelativistic Potential Scattering," Few Body Syst. 49 (2011) 41 arXiv:1008.1718 [nucl-th]] . 